\title{
A SYNONYMIC GENERIC CHECKLIST OF THE EUMENINAE (HYMENOPTERA: VESPIDAE)*
}

\author{
By James M. CARPENTER \\ Museum of Comparative Zoology, Harvard University, \\ Cambridge, MA 02138
}

The present work is an extension of a similar list in Carpenter (1983), and arose from preparatory work for a phylogenetic analysis of nearctic potter wasp genera (Carpenter and Cumming, 1985). The most recent available world list of genera is over 80 years old (Dalla Torre, 1904), and fully $57 \%$ of the genus-group names currently used in the Eumeninae have been proposed since Bluethgen (1938; for more detail on the history of eumenine taxonomy see Carpenter and Cumming, 1985). The following checklist includes all the currently recognized genera of Eumeninae sensu Carpenter (1981), with their synonyms and subgenera. The arrangement is alphabetical based upon most recent usage, and incorporates the decisions pertaining to eumenine generic nomenclature rendered by the International Commission on Zoological Nomenclature (ICZN) in Opinions 747 (1965), 893 (1970) and 1363 (1985). The format is basically that of Krombein et al. (1979). The original citations are followed by the type species designation. Synonyms, and subgenera with their citations and synonyms, are listed after this; the nominotypical subgenera are not listed separately. Where two dates are listed, the first is the true date of publication, whereas the date listed in parentheses is that printed on the paper. A misspelling is indicated by (!), and quotation marks are used for incorrect names. No effort has been made to list all misspellings; only those occurring in works considered important. Nomenclatural changes derive from ongoing work on a catalog of neotropical eumeninae (with J. van der Vecht) and a generic reclassification of this group: Neodiscoelius Stange is a junior objective synonym of Protodiscoelius Dalla Torre (new synonymy); Cephalastor Soika is raised to genus (new status), and its type species, Hypalastoroides depressus Soika, synonymized with Odynerus relativus Fox. In addition, type species are designated for

*Manuscript received by the editor July 14, 1985 
Stenolabus Schulthess (junior subjective synonym of Ischnocoelia Perkins) and Nesodynerus Perkins. These designations conform to standard generic concepts. Two nomina dubia. and four nomina nuda not otherwise placed are listed separately at the end of this paper.

It is not to be inferred that I agree with this classification, but considering the current confusion in eumenine taxonomy, a catalog of the available names and their status is a prerequisite for rectifying the situation.

Abispa Mitchell, 1838, Three. Exped. Interior Eastern Australia 1:104 (as subgenus of Vespa L.). Type species Abispa australiana Mitchell, 1838. Monotypic.

Abisba (!) Ashmead, 1902, Can. Ent. 34: 208 (gives as type Vespa ephippium Fabricius, 1775, originally not included). Monerebia Saussure, 1852, Ét. Fam. Vesp. 1: 98. Type species Odynerus splendidus Guerin, 1838. Designated by Vecht, 1960, Nova Guinea Zool. 6: 92.

Monorebia (!) Smith, 1857, Cat. Hym. Brit. Mus. 5: 42.

Monerobia (!) Bridwell, 1919, Proc. Hawaiian Entomol. Soc. 4: 120.

subg. Parabispa Vecht, 1960, Nova Guinea Zool. 10(6): 93, 94.

Type species Pterochilus eximius Smith, 1865. Original designation.

Acanthodynerus Gusenleitner, 1969, Boll. Mus. Civ. Ven. 19: 13. Type species Acanthodynerus giordanii Gusenleitner, 1969. Original designation.

Acarepipona Soika, 1985 (1983), Boll. Mus. Civ. Ven. 34: 189, 192. Type species Acarepipona insolita Soika, 1985. Original designation.

Acarodynerus Soika, 1962 (1961), Boll. Mus. Civ. Ven. 14: 64, 146. Type species Odynerus clypeatus Saussure, 1853. Original designation.

Acarozumia Bequaert, 1921, Rev. Zool. Afr. 9: 249 (as subgenus of Montezumia Saussure). Type species Nortonia amaliae Saussure, 1869. Monotypic.

Afrepipona Soika, 1965, Boll. Soc. Entomol. Ital. 95: 46. Type species Odynerus macrocephalus Gribodo, 1894. Original designation. 
Afreumenes Bequaert, 1926, Ann. S. Afr. Mus. 23: 486 (as subgenus of Eumenes Latreille). Type species Eumenes melanosoma Saussure, 1852. Monotypic.

Afrodynerus Soika, 1934, Ann. Mus. Civ. Genova 57: 25, 26 (as subgenus of Odynerus Latreille). Type species Odynerus monstruosus Soika, 1934. Monotypic.

Afroxanthodynerus Soika, 1979, Boll. Mus. Civ. Ven. 30: 243. Type species Afroxanthodynerus nigeriensis Soika, 1979. Original designation.

Alastor Lepeletier, 1841, Hist. Nat. Ins. Hym. 2: 668. Type species Alastor atropos Lepeletier, 1841. Designated by Ashmead, 1902, Can. Ent. 34: 210.

Antalastor Saussure, 1856, Ét. Fam. Vesp. 3: 328 (as division of Alastor). Type species Alastor atropos Lepeletier, 1841. Designated by ICZN, Opinion 893, 1970: 187.

Eualastor Dalla Torre, 1904, Gen. Ins. 19: 60. New name.

Belalastor Atanassov, 1967, Izv. Zool. Inst. Sof. 23: 167 (as subgenus of Alastor). Type species Alastor bulgaricus Atanassov, 1967 (= Alastor seidenstueckeri Bluethgen, 1956). Original designation.

subg. Parastalor Bluethgen, 1939, Veroeff. Dts. Kolon. Uebersee-

Mus. Bremen 2: 264. Type species Alastor algeriensis Bluethgen, 1939. Monotypic.

subg. Megalastor Bluethgen, 1951, Mitt. Muench. Entomol. Ges. 41: 169. Type species Alastor savignyi Saussure, 1852. Original designation.

Alastoroides Saussure, 1856, Ét. Fam. Vesp. 3: 327 (as subgenus of Alastor Lepeletier). Type species Alastor clotho Lepeletier, 1841. Designated by Ashmead, 1902, Can. Ent. 34: 210.

Paralastoroides Saussure, 1856, Ét. Fam. Vesp. 3: 328 (as division of subgenus Alastoroides Saussure of genus Alastor Lepeletier). Type species Alastor clotho Lepeletier, 1841. Monotypic. Rejected by ICZN, Opinion 893, 1970: 188, in favor of Alastoroides.

Alasteroides (!) Zavattari, 1912, Arch. Naturgesch. 78A(4): 255.

Alastorynerus Bluethgen, 1938 (1937), Konowia 16: 294. Type species Odynerus ludendorffi Dusmet, 1917. Original designation. 
Alastodynerus (!) Parker, 1966, Misc. Publ. Entomol. Soc. Am. 5: 157.

Alfieria Soika, 1934, Bull. Soc. Entomol. Egypte 18: 436. Type species Eumenes anomalus Zavattari, 1909. Original designation.

Alferia (!) Neave, 1939, Nomencl. Zool. 1: 111.

Allodynerus Bluethgen, 1938 (1937), Konowia 16: 280 (as subgenus Saussure, 1853. Original designation. (as "Lionotus floricola Sauss. 1852").

Delphinaloides Moczar, 1937, Folia Ent. Hung. 3: 15. Invalid; no type designated. Made available by Bohart, 1951, in Muesebeck et al., Cat. Hym. N. Am.: 888; with type species Odynerus delphinalis Giraud, 1866.

Allorhynchium Vecht, 1963, Zool. Verh. (Leiden) 60: 57, 58. Type species Vespa argentata Fabricius, 1804. Original designation.

Alphamenes Vecht, 1977, Proc. K. Ned. Akad. Wet. (C) 80: 238, 242. Type species Eumenes campanulatus Fabricius, 1804. Original designation.

Alphamenes Bertoni, 1934, Rev. Soc. Cient. Paraguary 3: 109 (as subgenus of Eumenes Latreille). Invalid; no type designated.

Ancistroceroides Saussure, 1855, Et́. Fam. Vesp. 3: 221 (as division of subgenus Ancistrocerus Wesmael of genus Odynerus Latreille; validated by ICZN, Opinion 893, 1970: 187. Type species Odynerus alastoroides Saussure, 1853. Designated by ICZN, Opinion 1363, 1985: 353.

Ancistrocerus Wesmael, 1836, Bull. Acad. Sci. Bruxelles 3: 45 (as subgenus of Odynerus Latreille). Type species Vespa parietum L., 1758. Designated by Girard, 1879, Traité Élém. Ent. 2(2): 900.

Aucistrocerus (!) Rudow, 1876, Arch. Ver. Freunde Naturgesch. Mecklenb. 30: 197.

Ancystrocerus (!) Dalla Torre, 1894, Cat. Hym. 9: $50 \mathrm{ff}$.

Euancistrocerus Dalla Torre, 1904, Gen. Ins. 19: 36. New name.

Antamenes Soika, 1958 (1957), Boll. Mus. Civ. Ven. 10: 214. Type species Odynerus flavocinctus Smith, 1857 (= Odynerus vernalis Saussure, 1853). Original designation.

subg. Australochilus Soika, 1962 (1961), Boll. Mus. Civ. Ven. 
14: 184. Invalid; no type designated. Made available by Soika, 1974 (1973), Boll. Mus. Civ. Ven. 24: 53; with type species Odynerus citreocinctus Saussure, 1867.

Antepipona Saussure, 1855, É́. Fam. Vesp. 3: 244 (as division of subgenus Odynerus of genus Odynerus Latreille; validated by ICZN, Opinion 893, 1970: 187). Type species Odynerus silaos Saussure, 1853. Designated by ICZN, Opinion 893, 1970: 187. Antepiponus Saussure, 1875, Smiths. Misc. Coll. 254: xxxv, 361. Emendation.

Antepipone (!) Dalla Torre, 1894, Cat. Hym. 9: 50, 96.

Mehelyella Moczar, 1937, Folia Ent. Hung. 3: 16. Invalid; no type designated. Made available by Bohart, 1951, in Muesebeck et al., Cat. Hym. N. Am.: 888; with type species Odynerus parvulus Lepeletier, 1841.

Odontodynerus Bluethgen, 1938 (1937), Konowia 16: 280 (as subgenus of "Euodynerus Bluethgen"). Type species Odynerus orbitalis Herrich-Schaeffer, 1841. Original designation.

Dichodynerus Bluethgen, 1938. Dts. Entomol. Z.: 444. Type species Odynerus vagabundus Dalla Torre, 1889. Original designation (as "Lionotus vagus Radoszkowsi (= vagabundus Dalla Torre nom. nov.)").

Metastenancistrocerus Bluethgen, 1938, Dts. Entomol. Z.: 460. Error for Dichodynerus; $c f$. Bluethgen, 1939, Veroeff. Dts. Kolon. Uebersee-Mus. Bremen 2: 246.

Anterhynchium Saussure, 1863, Mém. Soc. Phys. Hist. Nat. Genève 17: 205 (as division of Rhynchium Spinola). Type species $R y g$ chium synagroides Saussure, 1852. Designated by Vecht, 1963, Zool. Verh. (Leiden) 60: 73.

Anterrhynchium (!) Dalla Torre, 1904, Gen. Ins. 19: 33.

subg. Dirhynchium Vecht, 1963, Zool. Verh. (Leiden) 60: 74, 77. Type species Ancistrocerus flavopunctatus Smith, 1852. Original designation.

Antezumia Saussure, 1875, Smiths. Misc. Coll. 254: 113 (as division of Montzumia Saussure). Type species Montezumia chalybea Saussure, 1855. Designated by Bequaert, 1921, Rev. Zool. Afric. 9: 240.

Pinta Zavattari, 1912, Arch. Naturgesch. 78A(4): 6, 151. Type species Montezumia chalybea Saussure, 1855. Original designation. 
Antodynerus Saussure, 1855, Ét. Fam. Vesp. 3: 242, 287 (as division of subgenus Odynerus of genus Odynerus Latreille; validated by ICZN, Opinion 893, 1970: 187). Type species Vespa flavescens Fabricius, 1775 (“Odynerus punctum (Fabricius)" sensu Saussure, 1853). Designated by ICZN, Opinion 893, 1970: 187. Kalliepipona Soika, 1952 (1951), Riv. Biol. Colon. 11: 81 (as subgenus of Pseudepipona Saussure). Type species Rhynchium radiale Saussure, 1855 (as "Odynerus radialis"). Original designation.

Pseudokalliepipona Soika, 1955, Ann. Mus. R. Congo Belge Tervuren, Zool. 36: 366 (as subgenus of Pseudepipona Saussure, 1853. Type species Odynerus bellatulus Saussure, 1853. Original designation.

Parepipona Soika, 1957, Brit. Mus. (Nat. Hist.) Exped. S. W. Arabia 1(31): 477 (as subgenus of Pseudepipona Saussure). Type species Rhynchium radiale Saussure, 1855 (as "Odynerus radialis'). Original designation.

Anthodynerus (!) Soika, 1961, South Afr. Anim. Life 8: 445.

Araucodynerus Willink, 1968 (1967), Acta Zool. Lilloana 22: 143, 152. Type species Odynerus tuberculatus Saussure, 1853. Original designation.

Argentozethus Stange, 1979, Acta Zool. Lilloana 35: 729. Type species Argentozethus willinki Stange, 1979. Original designation.

Asiodynerus Kurzenko, 1977, Ins. Mongolia 5: 557. Type species Odynerus lucifer Kostylev, 1937. Original designation.

Astalor Schulthess, 1925, Konowia 4: 59, 207 (as subgenus of Alastor Lepeletier). Type species Astalor maidli Schulthess, 1925. Monotypic.

Astator (!) Schulthess, 1925, Konowia 4: 208.

Australodynerus Soika, 1962 (1961), Boll. Mus. Civ. Ven. 14: 65, 114. Type species Odynerus pusillus Saussure, 1856. Original designation.

Australozethus Soika, 1969, Boll. Mus. Civ. Ven. 19: 27, 29. Type species Australozethus tasmaniensis Soika, 1969. Original designation.

Bidentodynerus Soika, 1977 (1976), Mem. Soc. Entomol. Ital. 55: 177. Type species Odynerus bicolor Saussure, 1855. Original designation.

Brachymenes Soika, 1961, Verh. XI Int. Kongr. Entomol. Wien: 243. Type species Eumenes wagnerianus Saussure, 1875. Original designation. 
Brachyodynerus Bluethgen, 1938, Dts. Entomol. Z.: 450, 459. Type species Odynerus magnificus Morawitz, 1867. Original designation.

Brachypipona Gusenleitner, 1967, Polskie Pismo Ent. 37: 671. Type species Pseudepipona schmidti Gusenleitner, 1967. Original designation.

Desertodynerus Kurzenko, 1977, Zool. Zh. 56(6): 957. Type species Desertodynerus gratus Kurzenko, 1977. Original designation.

Calligaster Saussure, 1852, Ét. Fam. Vesp. 1: 22. Type species Calligaster cyanopterus Saussure, 1852. Designated by Ashmead, 1902, Can. Ent. 34: 205.

Cephalastor Soika, 1982 (1981), Boll. Mus. Civ. Ven. 32: 33, 40 (as subgenus of Hypalastoroides Saussure); NEW STATUS. Type species Hypalastoroides depressus Soika, 1969 (= Odynerus relativus Fox, 1902; NEW SYNONYMY). Original designation.

Cephalochilus Bluethgen, 1939, Mitt. Entomol. Ges. Halle 17: 13. Type species Pterochilus grandis Lepeletier, 1841 (=Vespa labiata Fabricius 1798). Original designation.

Cephalodynerus Parker, 1965, Ann. Entomol. Soc. Am. 58: 364. Type species Cephalodynerus unicornis Parker, 1965. Original designation.

Chelodynerus Perkins, 1902, Trans. Entomol. Soc. Lond.: 136. Type species Odynerus chelifer Perkins, 1899. Monotypic.

Chlorodynerus Bluethgen, 1951, Boll. Soc. Entomol. Ital. 81: 67, 75 (as subgenus of "Euodynerus Bluethgen"). Type species Odynerus chloroticus Spinola, 1838. Original designation.

Coeleumenes Vecht, 1963, Zool. Verh. (Leiden) 60: 16, 45. Type species Montezumia impavida Bingham, 1897. Original designation.

Ctenochilus Saussure, 1856, Ét. Fam. Vesp. 3: 323 (as division of Pterochilus (!) Klug). Type species Epipona pilipalpa Spinola, 1851. Monotypic.

Cuyodynerus Willink, 1968 (1967), Acta Zool. Lilloana 22: 143, 151. Type species Odynerus cuyanus Brèthes, 1903. Original designation.

Cyphodynerus Vecht, 1971, Entomol. Ber. (Amst.) 31: 127. Type species Odynerus dimidiatus Spinola, 1838 (non Odynerus dimidiatus Guérin, 1834; = Odynerus canaliculatus Saussure, 1855). Original designation. 
Cyphomenes Soika, 1978, Boll. Mus. Civ. Ven. 29: 13, 210. Type species Eumenes infernalis Saussure, 1875. Original designation.

Cyrtolabulus Vecht, 1969, Entomol. Ber. (Amst.) 29: 1. New name for Cyrtolabus Vecht.

Cyrtolabus Vecht, 1963, Zool. Verh. (Leiden) 60: 11 non Cyrtolabus Voss, 1925. Type species Cyrtolabus suavis Vecht, 1963. Original designation.

Delta Saussure, 1855, Ét. Fam. Vesp. 3: 130, 143 (as division of Eumenes Latreille). Type species Vespa maxillosa DeGeer, 1773 (= Vespa emarginata L., 1758). Designated by Bequaert, 1925, Bull. Brook. Entomol. Soc. 20: 137 (as "Sphex maxillosus').

Phi Saussure, 1855, Ét. Fam. Vesp. 3: 132 (as division of Eumenes Latreille) non Phi Saussure, 1854. Type species Vespa arcuata Fabricius, 1775. Designated by Bequaert, 1926, Ann. S. Afr. Mus. 23: 487.

Erinys Zirngiebl, 1953, Mitt. Pollichia (3)1: 173 non Erinys Rye, 1876. Type species Vespa unguiculata Villers, 1789. Monotypic.

Deuterodiscoelius Dalla Torre, 1904, Gen. Ins. 19: 18 (as division of Discoelius Latreille). Type species Odynerus verrauxii Saussure, 1852. Monotypic.

Pseudozethus Perkins, 1914, Pr. Zool. Soc. Lond.: 622. Type species Pseudozethus australensis Perkins, 1914 (= Odynerus verrauxii Saussure, 1852). Monotypic.

Diemodynerus Soika, 1962 (1961), Boll. Mus. Civ. Ven. 14: 65, 141. Type species Odynerus diemensis Saussure, 1853. Original designation.

Discoelius Latreille, 1809, Gen. Crust. Ins. 4: 140 (as subgenus of Eumenes Latreille). Type species Vespa zonalis Panzer, 1801. Monotypic.

Discaelius (!) Leach, 1815, Edinburgh Encyc. 9: 153.

Discaelias (!) Leach, 1815, Edinburgh Encyc. 9: 166.

Dicoelius (!) Haliday, 1836, Trans. Linn. Soc. Lond. 17: 325.

Discollius (!) Froggatt, 1892, Proc. Linn. Soc. N.S.W. 2(7): 226.

Tritodiscoelius Dalla Torre, 1904, Gen. Ins. 19: 18 (as division of Discoelius). Type species Vespa zonalis Panzer, 1801. 
Designated by Bequaert and Ruiz, 1942 (1940), Rev. Chil. Hist. Nat. 64: 217.

Dolichodynerus Bohart, 1939, Pan-Pac. Ent. 15: 97, 101 (as subgenus of Odynerus Latreille). Type species Odynerus turgiceps Bohart, 1939. Original designation.

Ectopioglossa Perkins, 1912, Ann. Mag. Nat. Hist. (8)9: 118. Type species Ectopioglossa australensis Perkins, 1912 (non Eumenes australensis Meade-Waldo, 1910; = Ectopioglossa polita australensis (Meade-Waldo)). Monotypic.

Elimus Saussure, 1852, Ét. Fam. Vesp. 1: 7. Type species Elimus australis Saussure, 1852. Monotypic.

Elisella Soika, 1974 (1972), Boll. Mus. Civ. Ven. 25: 109, 132. Type species Ellisella linae Soika, 1974. Original designation.

Epiodynerus Soika, 1958 (1957), Boll. Mus. Civ. Ven. 10: 195 (as subgenus of Pseudepipona Saussure). Type species Odynerus alecto Lepeletier, 1841. Original designation.

Epsilon Saussure, 1855, Ét. Fam. Vesp. 3: 229 (as division of subgenus Odynerus of genus Odynerus Latreille; validated by ICZN, Opinion 893, 1970: 187). Type species Odynerus dyscherus Saussure, 1852. Designated by ICZN, Opinion 893, 1970: 187.

Eudiscoelius Friese, 1904, Z. Hym. Dipt. 4: 16. Type species Eudiscoelius metallicus Friese, 1904. Monotypic.

Euchalcomenes Turner, 1908, Trans. Entomol. Soc. Lond.: 90.

Type species Euchalcomenes gilberti Turner, 1908. Original designation.

Eumenes Latreille, 1802, Hist. Nat. Crust. Ins. 3: 360. Type species Vespa coarctata L., 1758. Designated by Latreille, 1810, Consid. Gén. Crust. Arach. Ins.: 328.

Alpha Saussure, 1855, Ét. Fam. Vesp. 3: 128, 137 (as division of Eumenes) non Alpha Saussure, 1854. Type species Vespa coarctata L., 1758. Designated by Bequaert, 1926, Ann. S. Afr. Mus. 23: 435.

Eumenis Kriechbaumer, 1879, Entomol. Nachr. 5: 57. Emendation.

Eumenidion Schulthess, 1913, Soc. Entomol. 28: 2 (as subgenus). Type species Vespa coarctata L., 1758. Original designation.

Eumenidium (!) Sharp, 1915, Zool. Rec. Ins. 1913: 275.

subg. Zeteumenoides Soika, 1972, Boll. Soc. Entomol. Ital. 
104: 110 (as genus). Type species Eumenes filiformis Saussure, $1855(=$ Eumenes versicolor filiformis $)$. Original designation.

Eumenidiopsis Soika, 1939 (1938), Mem. Soc. Entomol. Ital. 17: 87 (as subgenus of Leptomenes Soika). Type species Leptomenes subtilis Soika, 1939. Original designation.

Eumicrodynerus Gusenleitner, 1972, Nachrbl. Bayer. Ent. 21: 74 (as subgenus of Microdynerus Thomson). Type species Leptomenes europaeus Soika, 1942. Original designation.

Euodynerus Dalla Torre, 1904, Gen. Ins. 19: 38 (as section of subgenus "Lionotus" Thomson of genus Odynerus Latreille; validated by ICZN, Opinion 893, 1970: 187. Type species Vespa dantici Rossi, 1790. Designated by Bluethgen, 1938 (1937), Konowia 16: 277.

subg. Pareuodynerus Bluethgen, 1938 (1937), Konowia 16: 278

(as subgenus of "Euodynerus Bluethgen"). Type species Vespa notata Jurine, 1807. Original designation.

Leionotus Saussure, 1851, Ét. Fam. Vesp. 1: 121 (as subgenus of Odynerus Latreille), non Leionotus Kirby and Spence, 1828. Type species Odynerus foraminatus Saussure, 1853. Designated by Bohart, 1951, in Muesebeck et al., Cat. Hym. N. Am.: 887.

Lionotus (!) Thomson, 1874, Opusc. Ent. 2: 85, non Lionotus Agassiz, 1846.

Lejonotus (!) Costa, 1882, Atti. R. Acad. Sci. Fis. Mat. Napoli 9: 37.

Eustenancistrocerus Bluethgen, 1938, Dts. Entomol. Z.: 443, 460 (as subgenus of "Stenancistrocerus Saussure" sensu Bluethgen, 1938). Type species Odynerus blanchardianus Saussure, 1855. Original designation.

subg. Hemistenancistrocerus Bluethgen, 1938, Dts. Entomol.

Z.: 443, 459 (as subgenus of "Stenancistrocerus Saussure" sensu Bluethgen, 1938). Type species Leptochilus parvulus Saussure, 1853 (non Odynerus parvulus Herrich-Schaeffer, 1938; = Odynerus pharao Saussure, 1863). Original designation.

subg. Parastenancistrocerus Bluethgen, 1938, Dts. Entomol. Z.: 444, 460 (as subgenus of "Stenancistrocerus Saussure" sensu Bluethgen, 1938). Type species Odynerus transitorius Morawitz, 1867. Original designation. 
Flammodynerus Soika, 1962 (1961), Boll. Mus. Civ. Ven. 14: 65, 124. Type species Odynerus subalaris Saussure, 1855. Original designation.

Gamma Zavattari, 1912, Arch. Naturgesch. 78A(4): 85 (as division of Eumenes Latreille). Type species Pachymenes ventricosa Saussure, 1852. Designated by Bequaert, 1926, Ann. S. Afr. Mus. 23: 486.

Gastrodynerus Bohart, 1984, Pan-Pac. Ent. 60: 12. Type species Stenodynerus vanduzeei Bohart, 1948. Original designation.

Gioiella Soika, 1985 (1983), Boll. Mus. Civ. Venezia 34: 30, 155. Type species Odynerus katonai Schulthess, 1913. Original designation.

Gribodia Zavattari, 1912, Arch. Naturgesch. 78A(4): 161. Type species Monobia cavifrons Gribodo, 1891 (= Odynerus confluentus Smith, 1857). Original designation.

Gymnomerus Bluethgen, 1938 (1937), Konowia 16: 286 (as subgenus of "Hoplomerus (Westwood) Agassiz" sensu Bluethgen, 1938). Type species Odynerus laevipes Shuckard, 1837. Original designation.

Hemipterochilus Ferton, 1909 (1908), Ann. Soc. Entomol. Fr. 77: 572 (as subgenus of Pterocheilus Klug). Type species Odynerus terricola Mocsary, 1883 (= Hemipterochilus bembeciformis terricola). Monotypic.

Pseudopterochilus Kostylev, 1940, Bull. Soc. Nat. Moscou,

Biol. (N.S.) 49: 153. Invalid; no type designated. Made available by Vecht, 1972, in Vecht and Fischer, Hym. Cat. 8: 19; with type species Odynerus bembeciformis Morawitz, 1867.

Hypalastoroides Saussure, 1856, Ét. Fam. Vesp. 3: 328 (as division of subgenus Alastoroides Saussure of genus Alastor Lepeletier; validated by ICZN, Opinion 893, 1970: 187). Type species Alastor brasiliensis Saussure, 1856. Monotypic.

Hypalastor Saussure, 1856, Ét. Fam. Vesp. 3: 328 (as division of subgenus Alastor of genus Alastor Lepeletier; validated by ICZN, Opinion 893, 1970: 187). Type species Odynerus angulicollis Spinola, 1851. Designated by ICZN, Opinion 893, 1970: 187. Rejected by Soika, 1960 (1958) Boll. Mus. Civ. Ven. 11: 35, acting as first reviser, in favor of Hypalastoroides.

Hypalasteroides (!) Zavattari, 1912, Arch. Naturgesch. 78A(4): 253. 
subg. Larastoroides Soika, 1982 (1981), Boll. Mus. Civ. Ven. 32: 33, 40. Type species Hypalastoroides costaricensis Soika, 1960. Original designation.

subg. Ortalastoroides Soika, 1982 (1981), Boll. Mus. Civ. Ven.

32: 34, 56. Type species Alastor singularis Saussure, 1852.

Original designation.

Ortastoroides (!) Soika, 1982 (1981), Boll. Mus. Civ. Ven. 32:

57.

Hypancistrocerus Saussure, 1855, Ét. Fam. Vesp. 3: 222 (as division of subgenus Ancistrocerus Wesmael of genus Odynerus Latreille; validated by ICZN, Opinion 893, 1970: 187). Type species Odynerus advena Saussure, 1855. Monotypic.

Hypancistroceroides (!) Saussure, 1856, Ét. Fam. Vesp. 3, Table des Matières: 8.

Hypancystrocerus (!) Dalla Torre, 1894, Cat. Hym. 9: 50.

Hypodynerus Saussure, 1855, Ét. Fam. Vesp. 3: 225 (as division of subgenus Odynerus of genus Odynerus Latreille; validated by ICZN, Opinion 893, 1970: 187). Type species Odynerus humeralis Haliday, 1836. Designated by Bequaert and Ruiz, 1943 (1941), Rev. Chil. Hist. Nat. 45: 69.

Hypodernus (!) Cameron, 1908, Trans. Am. Entomol. Soc.

34: 199.

Incodynerus Willink, 1968 (1967), Acta Zool. Lilloana 22: 143, 148. Type species Odynerus romandinus Saussure, 1853. Original designation.

Ischnocoelia Perkins, 1908, Proc. Hawaiian Entomol. Soc. 2: 28, 32. Type species Ischnocoelia xanthochroma Perkins, 1908. Monotypic.

Stenolabus Schulthess, 1910, Dts. Entomol. Z.: 189. Type species Stenolabus fulvus Schulthess, 1910. By present designation.

Ischnogasteroides Magretti, 1884 (1883), Boll. Soc. Entomol. Ital. 15: 251; 1884, Ann. Mus. Civ. Stor. Nat. Genova 21: 603. Type species Ischnogasteroides flavus Magretti, 1884 (= Ischnogasteroides leptogaster flavus). Monotypic.

Jucancistrocerus Bluethgen, 1938, Dts. Entomol. Z.: 442, 460 (as subgenus of "Stenancistrocerus Saussure" sensu Bluethgen, 1938). Type species Odynerus jucundus Mocsary, 1883. Original designation. 
Iucancistrocerus (!) Bluethgen, 1951, Mitt. Muench. Entomol. Ges. 41: 174.

subg. Eremodynerus Bluethgen, 1939, Veroeff. Dts. Kolon. Uebersee-Mus. Bremen 2: 257 (as genus). Type species Odynerus saharensis Soika, 1934. Original designation.

Katamenes Meade-Waldo, 1910, Ann. Mag. Nat. Hist. (8)5: 46. Type species Katamenes watsoni Meade-Waldo, 1910. Monotypic.

Knemodynerus Bluethgen, 1940, Entomol. Tidskr. 61:43 (as subgenus of "Euodynerus Bluethgen"). Type species Odynerus excellens Pérez, 1907. Original designation.

Labochilus Bluethgen, 1939, Mitt. Entomol. Ges. Halle 17: 12. Type species Pterochilus linguarius Saunders, 1905. Monotypic.

Leptopterocheilus Soika, 1953 (1952), Bull. Soc. Sci. Nat. Phys. Maroc. 32: 262. Type species Pterochilus linguarius Saunders, 1905. Original designation.

Labus Saussure, 1867, Zool. Novara 2, Hym.: 3. Type species Labus spiniger Saussure, 1867. Designated by Bingham, 1897, Fauna Brit. India Hym. 1: 348.

Laevimenes Soika, 1978, Boll. Mus. Civ. Ven. 29: 11, 359. Type species Eumenes laevigatus Brèthes, 1906. Original designation.

Leptochiloides Bohart, 1940, Ann. Entomol. Soc. Am. 33: 165. Type species Leptochiloides utahensis Bohart, 1940. Original designation.

Leptochilus Saussure, 1853, Ét. Fam. Vesp. 1: 233. Type species Pterochilus mauritanicus Lepeletier, 1841. Designated by Ashmead, 1902, Can. Ent. 34: 209 (as “mauritianus”!).

Zendalia Robertson, 1928, Flowers and Insects: 12. Type species Odynerus zendaloides Robertson, 1928 (= Leptochilus republicanus Dalla Torre, 1889). Designated by Bohart, 1951, in Muesebeck et al, Cat. Hym. N. Am.: 897.

subg. Euleptochilus Bluethgen, 1943, in Berland, Bull. Mus.

Hist. Nat. Paris (2)15: 316. Type species Odynerus oraniensis Lepeletier, 1841. Original designation.

subg. Lionotulus Bluethgen, 1938 (1937), Konowia 16: 276.

Type species Odynerus alpestris Saussure, 1855. Original designation.

subg. Neoleptochilus Bluethgen, 1961, Abh. Dts. Akad. Wiss. 
Berl. (2): 66, 100. Type species "Leptochilus medanae (Gribodo i.l.) André" (= Odynerus medanae Gribodo, 1886). Original designation.

subg. Sarochilus Gusenleitner, 1970, Isr. J. Entomol. 5: 57.

Type species Leptochilus alterego Gusenleitner, 1970. Original designation.

Leptodynerus Bluethgen, 1938, Dts. Entomol., Z.: 448, 457. Type species Leptodynerus biskrensis Bluethgen, 1938. Original designation.

Leptomenes Soika, 1939 (1938), Mem. Soc. Entomol. Ital. 17: 87. Type species Pachymenes congensis Bequaert, 1918 (=Odynerus eumenoides Smith, 1857). Original designation.

Leptomenoides Soika, 1962 (1961), Boll. Mus. Civ. Ven. 14: 64, 171. Type species Leptomenoides placidior Soika, 1962. Original designation.

Leptomicrodynerus Soika, 1985, Lavori Soc. Ven. Sc. Nat. 10: 37. Type species Leptomicrodynerus tieshengi Soika, 1985. Original designation.

Leucodynerus Bohart, 1982, J. Kans. Entomol. Soc. 55: 442. Type species Odynerus congressus Viereck, 1908. Original designation.

Macrocalymma Perkins, 1908, Proc. Hawaiian Entomol. Soc. 2: 28, 31. Type species Macrocalymma smithianum Perkins, 1908. Monotypic.

Maricopodynerus Viereck, 1908, Trans. Am. Entomol. Soc. 33: 397 (as subgenus of Odynerus Latreille). Type species Odynerus maricoporum Viereck, 1908. Monotypic.

Micreumenes Ashmead, 1902, Can. Ent. 34: 208. Type species Micreumenes currei Ashmead, 1902 (in key). Monotypic.

Smithia Saussure, 1855, Rev. Mag. Zool. 7: 371 non Smithia

Edwards and Haime, 1851. Type species Smithia natalensis Saussure, 1855. Monotypic.

Hymenosmithia Dalla Torre, 1904, Gen. Ins. 19: 61. New name for Smithia Saussure.

Microdynerus Thomson, 1874, Hym. Scand. 3: 58. Type species Odynerus exilis Herrich-Schaeffer, 1839. Designated by Jones, 1937, Entomol. Mon. Mag. 73: 15.

subg. Pseudomicrodynerus Bluethgen, 1938 (1937), Konowia

16: 276. Type species Odynerus parvulus Herrich-Schaeffer, 1838. Original designation. 
Pachymicrodynerus Bluethgen, 1938, Dts. Entomol.: 447, 455 (as subgenus of Pseudomicrodynerus). Type species Pseudomicrodynerus eurasius Bluethgen, 1938. Original designation.

Pseudomycrodynerus (!) Gusenleitner, 1977, Linz. Biol. Beitr. 9: 138.

Minixi Soika, 1978, Boll. Mus. Civ. Ven. 29: 14, 367. Type species Eumenes mexicanus Saussure, 1857. Original designation.

Mitrodynerus Vecht, 1981, Proc. K. Ned. Akad. Wet. (C) 84: 444. Type species Mitrodynerus vitripennis Vecht, 1981. Monotypic.

Monobia Saussure, 1852, Ét. Fam. Vesp. 1: 94. Type species Vespa quadridens L., 1763. Designated by Ashmead, 1902, Can. Ent. 34: 210.

Triarthra Dalla Torre, 1904, Gen. Ins. 19: 28 (as group of Monobia), non Triarthra Ehrenberg, 1832. Type species Odynerus cyanipennis Guérin, 1830. Designated by Bequaert, 1940, Rev. Entomol. 11: 822.

Tetrathra Dalla Torre, 1904, Gen. Ins. 19: 28. Type species Vespa quadridens L., 1763. Designated by Bequaert, 1940, Rev. Entomol. 11: 822.

Tetrarthra (!) Bequaert, 1940, Rev. Entomol. 11: 822.

Monodynerus Gusenleitner, 1982, Entomofauna 3: 279. Type species Monodynerus insimilis Gusenleitner, 1982. Original designation.

Montezumia Saussure, 1852, Ét. fam. Vesp. 1: 87. Type species Montezumia rufidentata Saussure, 1852 (= Odynerus azurescens Spinola, 1851). Designated by Ashmead, 1902, Can. Ent. 34: 207.

Alpha Saussure, 1855, Ét. Fam. Vesp. 3: 160 (as division of Montezumia), non Alpha Saussure, 1854. Type species Montezumia rufidentata Saussure, 1952 (= Odynerus azurescens Spinola, 1851). Designated by Bohart, 1951, in Muesebeck et al., Cat. Hym. N. Am.: 885.

Beta Saussure, 1855, Ét. Fam. Vesp. 3: 162 (as division of Montezumia). Type species Montezumia morosa Saussure, 1852. Designated by Bequaert, 1921, Rev. Zool. Afric. 9: 240.

Metazumia Saussure, 1875, Smiths. Misc. Coll. 254: 114 (as division of Montezumia). Type species Montezumia huas- 
teca Saussure, 1857. Designated by Bequaert, 1921, Rev. Zool. Afric. 9: 240.

Eumontezumia Dalla Torre, 1904, Gen. Ins. 19: 27. New name. Nesodynerus Perkins, 1901, Entomol. Mon. Mag. 37: 267. Type species Odynerus rudolphi Dalla Torre, 1889. By present designation.

Nortozumia Vecht, 1937, Treubia 16: 263. Type species Zethus rufofemoratus Cameron, 1903. Original designation.

Odynerus Latreille, 1802, Hist. Nat. Crust. Ins. 3: 362. Type species Vespa spinipes L., 1758. Designated by Shuckard, 1837, Mag. Nat. Hist. (N. S.) 1: 494.

Odynera Illiger, 1807, Magaz. Insektenk. 6: 196. Emendation. Epipone Kirby and Spence, 1815, Introd. Entomol. 1: 340, non "epipone" Latreille 1802, a vernacular name. Type species Vespa spinipes L., 1758. Monotypic.

Oplopus Wesmael, 1836, Bull. Acad. Sci. Bruxelles 3: 45 (as subgenus of Odynerus), non Oplopus Laporte, 1832. Type species Vespa spinipes L., 1758. Designated by Girard, 1879, Traité Élém. Ent. 2(2): 902.

Oplomerus Westwood, 1840, Intro. Mod. Classif. Ins. 2(Synopsis): 84. New name for Oplopus Wesmael; non Oplomerus Dejean, 1833, a nomen nudum.

Hoplomerus Agassiz, 1846, Nomencl. Zool. Index Univ.: 185. Emendation of Oplomerus Westwood.

Hoplopus Agassiz, 1846, Nomencl. Zool. Index Univ.: 186. Emendation of Oplopus Wesmael, non Hoplopus D'Orbigny, 1838.

Epiponus Saussure, 1875, Smiths. Misc. Coll. 254: 360 (as subgenus of Odynerus Latreille). Emendation of "Epipona Shuckard" sensu Saussure, 1855, an incorrect spelling of Epipone Kirby and Spence.

Hoplonus (!) Dalla Torre, 1889, Ent. Almanach.: 11.

Euepipona Dalla Torre, 1904, Gen. Ins. 19: 39. New name for Epiponus Saussure. Type species Vespa spinipes L., 1758. Designated by Richards, 1937, Gen. Names Br. Ins. 5: 128. subg. Allogymnomerus Bluethgen, 1951, Mitt. Muench. Entomol. Ges. 41: 174 (as subgenus of Hoplomerus Westwood). Type species Odynerus consobrinus Dufour, 1839. Original designation. 
subg. Monoplomerus Bluethgen, 1941, Arch Naturgesch. (N. F.) 10: 308 (as subgenus of Hoplomerus Westwood). Type species Hoplomerus caroli Morawitz, 1885. Original designation.

subg. Spinicoxa Bluethgen, 1938 (1937), Konowia 16: 285 (as subgenus of "Hoplomerus (Westwood) Agassiz" sensu Bluethgen, 1938). Type species Vespa reniformis Gmelin, 1790. Original designation.

Omicroides Soika, 1935, Ann. Mus. Civ. Genova 57: 129 (as subgenus of Eumenes Latreille). Type species Eumenes singularis Smith, 1857. Original designation.

Omicron Saussure, 1855, Ét. Fam. Vesp. 3: 133, 148 (as division of Eumenes Latreille). Type species Zethus ? globicollis Spinola, 1841. Designated by Bequaert, 1926, Ann. S. Afr. Mus. 23: 486. Beta Saussure, 1875, Smiths. Misc. Coll. 254: 88 (as division of Eumenes Latreille) non Beta Saussure, 1855. Type species Eumenes nortonianus Saussure, 1875. Designated by Bequaert, 1926, Ann. S. Afr. Mus. 23: 486.

Amphimenes Bertoni, 1923, Rev. Soc. Cient. Paraguay 1: 53, non Amphimenes Bates, 1873. Type species Eumenes totonacus Saussure, 1875. Monotypic.

Onychopterocheilus Bluethgen, 1955, Mitt. Muench. Ges. 44/45: 407 (as subgenus of Pterocheilus). Type species Odynerus daw Dusmet, 1903. Original designation.

Orancistrocerus Vecht, 1963, Zool. Verh. (Leiden) 60: 58, 99. Type species Odynerus drewseni Saussure, 1857. Original designation.

Oreumenes Bequaert, 1926, Ann. S. Afr. Mus. 23: 488 (as subgenus of Eumenes Latreille). Type species Eumenes harmandi Pérez, 1905 (= Eumenes decoratus Smith, 1852). Original designation.

Oreumenoides Soika, 1961, Verh. XI Int. Kongr. Entomol. Wien: 245. Type species Eumenes edwardsi Saussure, 1852. Original designation.

Ovodynerus Soika, 1985 (1983), Boll. Mus. Civ. Ven. 34: 31, 130. Type species Odynerus capicola Meade Waldo, 1915. Original designation.

Pachodynerus Saussure, 1870, Rev. Mag. Zool. 22: 56 (as division of subgenus Odynerus of genus Odynerus Latreille; vali- 
dated by ICZN, Opinion 893, 1970: 187). Type species Odynerus californicus Saussure, 1870. Designated by Bohart, 1951, in Muesebeck et al., Cat. Hym. N. Am.: 892.

Pachyodynerus (!) Dalla Torre, 1894, Cat. Hym. 9: 82.

Monobiella Ashmead, 1900, Trans. Entomol. Soc. Lond.: 312

(as genus). Type species Vespa atrata Fabricius, 1798. Monotypic.

Pachyodernus (!) Cameron, 1908, Trans. Am. Entomol. Soc. 34: 199.

Pachycoelius Soika, 1969, Boll. Mus. Civ. Ven. 19: 28, 54. Type species Pachycoelius brevicornis Soika, 1969. Original designation.

Pachymenes Saussure, 1852, Ét. Fam. Vesp. 1: 73. Type species Pachymenes sericea Saussure, 1852. Designated by Ashmead, 1902, Can. Ent. 34: 208.

Pachimenes (!) Saussure, 1855, Ét. Fam. Vesp. 3: 153.

Pachyminixi Soika, 1978, Boll. Mus. Civ. Ven. 29: 14, 387. Type species Eumenes sumichrasti Saussure, 1875. Original designation.

Parachilus Soika, 1961 (1960), Atti Soc. Ital. Sci. Nat. 99: 389, 392. Type species Pterochilus capensis Saussure, 1854. Original designation.

Paragymnomerus Bluethgen, 1938 (1937), Konowia 16: 286 (as subgenus of "Hoplomerus (Westwood) Agassiz" sensu Bluethgen, 1938). Type species Odynerus spiricornis Spinola, 1808. Original designation.

Paralastor Saussure, 1856, Ét. Fam. Vesp. 3: 328 (as division of subgenus Alastor of genus Alastor Lepeletier; validated by ICZN Opinion 893, 1970: 187). Type species Alastor tuberculatus Saussure, 1853, Designated by ICZN, Opinion 893, 1970: 187.

Paraleptomenes Soika, 1970, Boll. Mus. Civ. Ven. 20/21: 79. Type species Paraleptomenes nurseanus Soika, 1970. Original designation.

Paralionotulus Bluethgen, 1938 (1937), Konowia 16: 293. Type species Leptochilus mervensis Radoszkowski, 1887. Original designation.

Pseudolionotulus (!) Bluethgen, 1938, Dts. Entomol. Z.: 446, 454. Type species Leptochilus mervensis Radoszkowski, 
1887. Original designation.

Paramischocyttarus Magretti, 1884 (1883) Boll. Soc. Entomol. Ital. 15: 250; 1884, Ann. Mus. Civ. Genova 21: 600. Type species Paramischocyttarus subtilis Magretti, 1884. Monotypic.

Tanyzethus Cameron, 1910, Wiss. Ergebn. Schwed. Zool.

Exped. Kilimandjaro (8)6: 195. Type species Tanyzethus africanus Cameron, 1910. Monotypic.

Parancistrocerus Bequaert, 1925, Trans. Am. Entomol. Soc. 51: 64 (as subgenus of Ancistrocerus Wesmael). Type species Odynerus fulvipes Saussure, 1855. Original designation.

Paranortonia Bequaert, 1940, Ann. Entomol. Soc. Am. 33: 100 (as subgenus of Pachymenes Saussure). Type species Nortonia tolteca Saussure, 1875. Original designation.

Paranortonia Bertoni, 1934, Rev. Soc. Cient. Paraguay 3: 109 (as genus). Invalid; no type designated.

Pararhaphidoglossa Schulthess, 1910, Dts. Entomol. Z.: 187. Type species Pararhaphidoglossa fulva Schulthess, 1910. Original designation.

Pararaphidoglossa (!) Zavattari, 1912, Arch. Naturgesch. 78A(4): 5; Soika, 1941, Boll. Soc. Ven. Stor. Nat. 2: 227 and 1978, Boll. Mus. Civ. Ven. 29.

Pararrhynchium Saussure, 1855, Ét. Fam. Vesp. 3: 173 (as division of Rhynchium Spinola). Type species Rhynchium ornatum Smith, 1852. Monotypic.

Prorhynchium Saussure, 1855, Ét. Fam. Vesp. 3: 174 (as division of Rhynchium Spinola). Type species Rhynchium smithii Saussure, 1855. Monotypic.

Prorrhynchium (!) Saussure, 1856, Ét. Fam. Vesp. 3: 348, Table des Matières: 8.

Pararhynchium (!) Saussure, 1862, Stett. Entomol. Ztg. 23: 182.

Parrhynchium (!) Dalla Torre, 1894, Cat. Hym. 9: 42.

Paravespa Radoszkowski, 1886, Hor. Soc. Entomol. Ross. 20: 46. Type species Hoplomerus komarowi Radoszkowski, 1886 (= Odynerus quadricolor Morawitz, 1885). Monotypic.

Theletor Kokujev, 1912, Izv. Kavkaz. Muz. 7: 4 (under description of Rhynchium caucasicum). Type species Rhynchium caucasicum Kokujev, 1912. Designated by Vecht, 1972, in Vecht and Fischer, Hym. Cat. 8: 4. 
subg. Gestrodynerus Soika, 1961 (1960), Atti Soc. Ital. Sci. Nat. 99: 361, 369. Type species Rygchium gestroi Magretti, 1884. Original designation.

Parazumia Saussure, 1855, Ét. Fam. Vesp. 3: 166 (as division of Montezumia Saussure). Type species Odynerus carinulatus Spinola, 1851. Designated by Bequaert, 1921, Rev. Zool. Afric. 9: 241.

Pareumenes Saussure, 1855, Ét. Fam. Vesp. 3: 133 (as division of Eumenes Latreille). Type species Eumenes quadrispinosus Saussure, 1855. Designated by Bequaert, 1918, Bull. Am. Mus. Nat. Hist. 39: 271.

subg. Nortonia Saussure, 1869, Stett. Entomol. Z. 30: 53 (as genus). Type species Odynerus intermedius Saussure, 1853. Original designation.

Notonia (!) Sonan, 1938, Arb. Morph. Tax. Ent. 5: 70.

Parifodynerus Soika, 1962 (1961), Boll. Mus. Civ. Ven. 14: 64, 167. Type species Parifodynerus parificus Soika, 1962. Original designation.

Parodontodynerus Bluethgen, 1938 (1937), Konowia 16: 280 (as subgenus of "Euodynerus" Bluethgen). Type species Eumenes ephippium Klug, 1817. Original designation.

Paradontodynerus (!) Guichard, 1978, Entomol. Gazette 31: 45.

Parodynerus Saussure, 1855, Ét. Fam. Vesp. 3: 245 (as division of subgenus Odynerus of genus Odynerus Latreille; validated by ICZN, Opinion 893, 1970: 187). Type species Vespa bicincta Fabricius, 1781. Designated by Soika, 1958 (1957), Boll. Mus. Civ. Ven. 10: 214.

Pirhosigma Soika, 1978, Boll. Mus. Civ. Ven. 29: 11, 229. Type species Eumenes simulans Saussure, 1875. Original designation.

Plagiolabra Schulthess, 1903 (March), Verh. Zool. Bot. Ges. Wien 53: 361, 365. Type species Plagiolabra nigra Schulthess, 1903. Monotypic.

Leontiniella Brèthes, 1903 (Sept.), An. Mus. Nac. Buenos Aires

(3)2: 265. Type species Leontiniella argentina Brèthes, 1903. Monotypic.

Postepipona Soika, 1974 (1972), Boll. Mus. Civ. Ven. 25: 77. Type species Postepipona socotrae Soika, 1974. Original designation. 
Proepipona Soika, 1977, Steenstrupia 4: 125, 126. Type species Vespa lateralis Fabricius, 1781. Original designation.

Protodiscoelius Dalla Torre, 1904, Gen. Ins. 19: 18 (as division of Discoelius Latreille). Type species "Epipona chilensis Spinola, 1851 = Discoelius merula Haliday, 1836". Designated by Bequaert and Ruiz, 1942 (1940), Rev. Chil. Hist. Nat. 64: 217. Neodiscoelius Stange, 1979, Acta Zool. Lilloana 35: 729; NEW

SYNONYMY. Type species Discoelius merula Haliday, 1836. Original designation.

Pseudabispa Vecht, 1960, Nova Guinea Zool. 10(6): 91, 102. Type species Odynerus abispoides Perkins, 1912. Original designation.

Pseudacaromenes Soika, 1978, Boll. Mus. Civ. Ven. 29: 15 (in key). Type species Eumenes alfkeni Ducke, 1904. Original designation.

Pseudoacaromenes (!) 1981, Zool. Record 115 (1978) Ins.: 262, List of new generic and subgeneric names: 15, 39.

Pseudalastor Soika, 1962 (1961), Boll. Mus. Civ. Ven. 14: 65, 131. Type species Odynerus concolor Saussure, 1853. Original designation.

Pseudepipona Saussure, 1856, Ét. Fam. Vesp. 3: 309 (as division of subgenus "Epipona" of genus Odynerus Latreille; validated by ICZN, Opinion 893, 1970: 187). Type species Odynerus herrichii Saussure, 1856. Monotypic.

Metepipona Bluethgen, 1951, Mitt. Muench. Entomol. Ges. 41:

193 (as subgenus of Pseudepipona). Type species Odynerus peculiaris Morawitz, 1895. Original designation.

Trichepipona Bluethgen, 1951, Mitt. Muench. Entomol. Ges. 41: 171, 193 (as subgenus of Pseudepipona). Type species Odynerus lativentris Saussure, 1855. Original designation.

Leptepipona Bluethgen, 1951, Mitt. Muench. Entomol. Ges. 41: 171, 194 (as subgenus of Pseudepipona). Type species Vespa tripunctata Fabricius, 1787. Original designation.

Pseudopipona (!) Opinion 893, ICZN, 1970, Bull. Zool. Nomencl. 26: 187.

Pseudepipone (!) Bytinski-Salz and Gusenleitner, 1971, Isr. J. Ent. 6: 298.

subg. Deuterepipona Bluethgen, 1951, Mitt. Muench. Entomol. Ges. 41: 171, 194 (as genus). Type species Odynerus ionius Saussure, 1855. Original designation. 
Pseudochilus Saussure, 1856, Ét. Fam. Vesp. 3: 321 (as division of "Pterochilus" Klug). Type species Pterochilus glabripalpis Saussure, 1852. Monotypic.

Pseudodontodynerus Bluethgen, 1939, Veroeff, Dts. Kolon. Uebersee-Mus. Bremen 2: 249. Type species Odynerus pretiosus Dusmet, 1928. Monotypic.

Pseudodynerus Saussure, 1855, Ét. Fam. Vesp. 3: 220 (as division of subgenus Ancistrocerus Wesmael of genus Odynerus Latreille; validated by ICZN, Opinion 893, 1970: 187). Type species Odynerus luctuosus Saussure, 1855. Monotypic.

Pseudoleptochilus Bluethgen, 1938 (1937), Konowia 16: 294. Type species Odynerus frenchi Dusmet, 1917. Original designation (as "Lionotus frenchi Dusmet").

Pseudonortonia Soika, 1936, Ann. Mus. Civ. Genova 59: 268. Type species Odynerus difformis Saussure, 1853. Original designation.

Subancistroceroides Bluethgen, 1938, Dts. Entomol. Z.: 441, 460 (as subgenus of "Subancistrocerus Sauss." sensu Bluethgen, 1938). Type species Odynerus aegyptiacus Saussure, 1863. Original designation.

Pseudopterocheilus Perkins, 1901, Entomol. Mon. Mag. 37: 266. Type species Odynerus pterocheiloides Perkins, 1899. Original designation.

Pseudopterochilus (!) Dalla Torre, 1904, Gen. Ins. 19: 39.

Pseudosymmorphus Bluethgen, 1938 (1937), Konowia 16: 293. Type species Odynerus hindenburgi Dusmet, 1917. Original designation.

Pseudozumia Saussure, 1875, Smiths. Misc. Coll. 254: 128 (as division of Montezumia Saussure). Type species Montezumia indica Saussure, 1855. Monotypic.

Pseudzumia (!) Dalla Torre, 1894, Cat. Hym. 9: 38.

Pseumenes Soika, 1935, Ann. Mus. Civ. Genova 57: 145 (as subgenus of Pareumenes Saussure). Type species Eumenes eximius Smith, 1861. Original designation.

Psiliglossa Saunders, 1872, Trans. R. Entomol. Soc. Lond.: 42. New name for Stenoglossa Saussure.

Stenoglossa Saussure, 1852, Ét.Fam. Vesp. 1: 4, non Stenoglossa Chaudoir, 1848. Type species Raphiglossa odyneroides Saunders, 1850. Monotypic.

Psiloglossa Dalla Torre, 1894, Cat. Hym. 9: 8. Emendation. 
Pterocheilus Klug, 1805, Beitr. Natuurk. 1: 143. Type species Vespa phalerata Panzer, 1797. Designated by Blanchard, 1840, in Laporte, Hist. Nat. Ins. 3: 389.

Pterochilus (!) Illiger, 1807, Mag. Insektenk. 6: 196.

Pterochile (!) Blanchard, 1840, in Laporte, Hist. Nat. Ins. 3: 389.

Pterochylus (!) Saussure, 1853, Ét.Fam. Vesp. 1: 239.

Odontopterochilus Kostylev, 1940, Bull. Soc. Nat. Moscou,

Biol. (N. S.) 49: 148 (as subgenus of Pterocheilus). Invalid; no type designated. Made available by Vecht, 1971, Entomol. Ber. (Amst.) 31: 127; with type species Pterocheilus heptneri Kostylev, 1940.

Nannopterochilus Bluethgen, 1961, Ab. Dts. Akad. Wiss. Berl. 1961: 62, 86, 231. Type species Vespa phalerata Panzer, 1797. Original designation.

subg. Megapterocheilus Bohart, 1940, Ann. Entomol. Soc. Am. 33: 169, 173. Type species Pterochilus mirandus Cresson, 1879. Original designation.

subg. Onchopterocheilus Bohart, 1940, Ann. Entomol. Soc. Am. 33: 169, 191. Type species Pterochilus comptus Cresson, 1879. Original designation.

subg. Micropterocheilus Bohart, 1940, Ann. Entomol. Soc. Am. 33: 168, 201. Type species Pterocheilus desertorum Bohart, 1940. Original designation.

Pteromenes Soika, 1961 (1960), Atti Soc. Ital. Sci. Nat. 99: 389, 407. Type species Pterochilus paradisiacus Soika, 1941. Original designation.

Raphiglossa Saunders, 1850, Trans. R. Entomol. Soc. Lond. (2)1: 71. Type species Raphiglossa eumenoides Saunders, 1850. Designated by Ashmead, 1902, Can. Ent. 34: 206.

Raphidoglossa Dalla Torre, 1894, Cat. Hym. 9: 7. Emendation.

Raphiglossoides Soika, 1936, Boll. Soc. Entomol. Ital. 68: 77. Type species Raphiglossoides aethiopicus Soika, 1936. Original designation.

Rhynchalastor Meade-Waldo, 1910, Ann. Mag. Nat. Hist. (8)6(31): 110. Type species Rhynchalastor fuscipennis Meade-Waldo, 1910. Monotypic.

Rhynchium Spinola, 1806, emendation of Rygchium Spinola, 1806; validated by ICZN, Opinion 747, 1965: 186. Type species $R y g$ - 
chium (!) europaeum Spinola, 1806 (= Vespa oculata Fabricius, 1781). Monotypic.

Rygchium Spinola, 1806, Ins. Ligur. 1: 84 incorrect original spelling for Rhynchium.

Rhynchium Billberg, 1820, Enum. Ins.: 109. Emendation of Rychium (!) Spinola.

Rynchium Sturm, 1829, Verz. Ins. Nurnberg: 12. Emendation.

Rhygchium Saussure, 1853, Ét. Fam. Vesp. 1: xxxi, 276. Emendation.

Rhynchuium (!) Saussure, 1863, Mém. Soc. Phy. Hist. Nat. Genève 17: 242.

Eurrhynchium Dalla Torre, 1904, Gen. Ins. 19: 33. New name. Rygohium (!) Willink, 1982, Bol. Ac. Nac. Sci. 55: 195.

Smeringodynerus Snelling, 1975, Proc. Entomol. Soc. Wash. 77: 56. Type species Odynerus morelios Saussure, 1857. Original designation.

Sphaeromenes Soika, 1978, Boll. Mus. Civ. Ven. 29: 12, 225. Type species Sphaeromenes discrepatus Soika, 1978. Original designation.

Spinilabochilus Kurzenko, 1981, Hym. Far East: 81, 97. Type species Spinilabochilus turcmenicus Kurzenko, 1981. Original designation.

Stellepipona Soika, 1974 (1973), Boll. Mis. Civ. Ven. 24: 106. Type species Odynerus stellenboschensis Cameron, 1905. Original designation.

Stenancistrocerus Saussure, 1863, Mém. Soc. Phys. Hist. Nat. Genève 17: 216 (as division of subgenus Ancistrocerus Wesmael of genus Odynerus Latreille; validated by ICZN, Opinion 893, 1970: 187). Type species Odynerus atropos Lepeletier, 1841. Designated by Bequaert, 1925, Trans. Am. Entomol. Soc. 51: 63 .

Stenancystrocerus (!) Dalla Torre, 1894, Cat. Hym. 9: 55-95. Atropancistrocerus Bluethgen, 1938, Dts. Entomol. Z.: 442, 444, 461. Type species Odynerus hispanicus Dusmet, 1903. Original designation.

subg. Paratropancistrocerus Bluethgen, 1938, Dts. Entomol. Z.: 442.461 (as subgenus of Atropancistrocerus). Type species Odynerus transcaspicus Kostylev, 1935. Original designation. 
Stenodyneriellus Soika, 1962 (1961), Boll. Mus. Civ. Ven. 14: 65, 71. Type species Stenodyneriellus turneriellus Soika, 1962. Original designation.

Stenodyneroides Soika, 1940, Ann. Mus. Civ. Genova 60: 471 (as subgenus of Odynerus Latreille). Type species Odynerus corvus Meade-Waldo, 1915. Original designation.

Stenodynerus Saussure, 1863, Mém. Soc. Phys. Hist. Nat. Genève 17: 228 (as division of subgenus Odynerus of genus Odynerus Latreille; validated by ICZN, Opinion 893, 1970: 187). Type species Odynerus chinensis Saussure, 1863. Designated by Bohart, 1939, Pan-Pac. Ent. 15: 100.

Stemodynerus (!) Rohwer, 1913, Proc. U.S. Nat. Mus. 44: 445. Nannodynerus Bluethgen, 1938 (1937), Konowia 16: 281 (as subgenus of "Euodynerus Bluethgen"). Type species Lionotus teutonicus Bluethgen, 1937. Original designation.

Parhypodynerus Soika, 1974 (1973), Boll. Mus. Civ. Ven. 24: 110. Type species Odynerus pavidus Kohl, 1905. Original designation.

Stenonartonia Soika, 1974 (1973), Boll. Mus. Civ. Ven. 24: 25. New name for Paranortonia Soika.

Paranortonia Soika, 1941, Boll. Soc. Ven. Stor. Nat. 2: 25 non Paranortonia Bequaert, 1940. Type species Nortonia polybioides Schulthess, 1904. Original designation.

Stenosigma Soika, 1978, Boll. Mus. Civ. Ven. 29: 14, 407. Type species Eumenes allegrus Zavattari, 1912. Original designation.

Stroudia Gribodo, 1892 (1891), Boll. Soc. Entomol. Ital. 23: 262. Type species Stroudia armata Gribodo, 1892. Monotypic.

Subancistrocerus Saussure, 1855, Ét. Fam. Vesp. 3: 206 (as division of subgenus Ancistrocerus Wesmael of genus Odynerus Latreille; validated by ICZN, Opinion 893, 1970: 187). Type species Odynerus sichelii Saussure, 1854. Designated by Bequaert, 1925, Trans. Am. Entomol. Soc. 51: 61.

Epancistrocerus Saussure, 1856, Ét. Fam. Vesp. 3: 352. New name for Subancistrocerus Saussure. Type species Odynerus sichelii Saussure, 1854. Designated by Bequaert, 1925, Trans. Am. Entomol. Soc. 51: 61.

Symmorphoides Soika, 1977 (1976), Boll. Mus. Civ. Ven. 28: 171, 172. Type species Symmorphoides maroccanus Soika, 1976. Original designation. 
Symmorphus Wesmael, 1836, Bull. Acad. Sci. Bruxelles 3: 45 (as subgenus of Odynerus Latreille). Type species Odynerus elegans Wesmael, 1833. Designated by Richards, 1935, Trans. R. Entomol. Soc. Lond. 83: 162.

Protodynerus Saussure, 1855, Ét. Fam. Vesp. 3: 184, 186. New name for Symmorphus.

Synomorphus (!) Rohwer, 1917, Proc. U.S. Nat. Mus. 53: 234.

"Odynerus Latreille" sensu Bluethgen, 1938 (1937), Konowia 16: 274, 291. Type species Vespa muraria L., 1758. Designated by Bluethgen, 1938 (1937), Konowia 16: 274, 291.

Koptodynerus Bluethgen, 1943, Stett. Entomol. Z. 104: 152 (as subgenus of "Odynerus Latreille" sensu Bluethgen). Type species Symmorphus declivus Harttig, 1932. Monotypic.

subg. Parasymmorphus Cumming and Vecht, 1986, Entomol. Ber. (Amst.) 46: 23. Type species Odynerus momunganensis Schulthess, 1934. Original designation.

Synagris Latreille, 1802, Hist. Nat. Crust. Ins. 3: 360. Type species Vespa cornuta L., 1758 (as Vespa cornuta F.). Monotypic.

Eusynagris Dalla Torre, 1904, Gen. Ins. 19: 30. New name for Synagris.

Catilostenus Meunier, 1888, Nat. Sicil. 7: 150. Type species Catilostenus nigroviolaceus Meunier, 1888. Monotypic. Identity doubtful.

subg. Paragris Saussure, 1855, Ét. Fam. Vesp. 3: 156 (as division of Synagris). Type species Synagris humberti Saussure, 1855. Designated by Ashmead, 1902, Can. Ent. 34: 210 (as $P$. hubertii!).

Hypagris Saussure, 1855, Ét. Fam. Vesp. 3: 157 (as division of Synagris). Type species Synagris abdominalis Saussure, 1855 (= Synagris analis Saussure, 1852). Designated by Ashmead, 1902, Can. Ent. 34: 210.

Antagris Saussure, 1863, Mém. Soc. Phys. Hist. Nat. Genève 17: 181 (as division of Synagris). Type species Synagris aequatorialis Saussure, 1852 (= Synagris spiniventris Illiger, 1802). Designated by Ashmead, 1902, Can. Ent. 34: 210.

subg. Pseudagris Saussure, 1863, Mém. Soc. Phys. Hist. Nat. Genève 17: 203 (as division of Synagris). Type species Synagris carinata Saussure, 1863. Monotypic. 
subg. Rhynchagris Maidl, 1914, Anz. Ak. Wiss. Wien 51: 91.

Type species Synagris vicaria Stadelmann, 1898. Monotypic.

Syneuodynerus Bluethgen, 1951, Boll. Soc. Entomol. Ital. 81: 67, 75

(as subgenus of "Euodynerus Bluethgen"). Type species Odynerus egregius Herrich-Schaeffer, 1839. Original designation. Syneodynerus (!) Kurzenko, 1981, Hym. Far East: 101.

Tachyancistrocerus Soika, 1952, Boll. Soc. Venez. Stor. Nat. 6: 37. New name for Subancistrocerus Bluethgen.

"Subancistrocerus (Saussure) nov. gen." Bluethgen 1938, Dts.

Entomol. Z: 441, 460 non Subancistrocerus Saussure, 1855.

Type species Odynerus rhodensis Saussure, 1855. Original designation.

Tachymenes Soika, 1983 (1982), Boll. Mus. Civ. Ven. 33: 118. Type species Odynerus vulneratus Saussure, 1855. Original designation.

Tricarinodynerus Soika, 1952 (1951), Riv. Biol. Colon. 11: 73, 79. Type species Odynerus guerinii Saussure, 1852. Original designation.

Carinodynerus Soika, 1957 Brit. Mus. (Nat. Hist.) Exped. S.

W. Arabia 1(31): 478 (as subgenus of Pseudepipona Saussure). Type species Odynerus guerinii Saussure, 1852. Original designation.

Tricomenes Soika, 1978, Boll. Mus. Civ. Ven. 29: 10, 254. Type species Eumenes pilosa Fox, 1899. Original designation.

Tropidodynerus Bluethgen, 1939, Veroeff, Dts. Kolon. UeberseeMus. Bremen 2(3): 259, 260. Type species Polistes interrupta Brullé, 1832. Original designation.

Xanthodynerus Bluethgen, 1954, Dts. Entomol. Z. (N. F.) 1: 255 (as subgenus of "Euodynerus Bluethgen"). Type species Odynerus octavus Soika, 1943. Original designation.

Xenorhynchium Vecht, 1963, Zool. Verh. (Leiden) 60: 111. Type species Vespa nitidula Fabricius, 1798. Original designation.

Zeta Saussure, 1855, Ét. Fam. Vesp. 3: 132, 146 (as division of Eumenes Latreille). Type species Sphex abdominalis Drury, 1770. Designated by Bequaert, 1926, Ann. S. Afr. Mus. 23: 487. Zeteumenes Bertoni, 1921, Rev. Soc. Cient. Paraguay 1: 117.

Type species Vespa canaliculata Olivier, 1791 (= Sphex argillacea L., 1758). Designated by Vecht, 1977, Proc. K. Ned. Akad. Weten. (C)80: 242. 
Zetamenes (!) Bertoni, 1926, Rev. Soc. Cient. Paraguay 2: 75. Beteumenes Bertoni, 1934, Rev. Soc. Cient. Paraguay 3: 109 (as subgenus of Zeteumenes). Invalid; no type designated.

Zetheumenidion Bequaert, 1926, Ann. S. Afr. Mus. 23: 487 (as subgenus of Eumenes Latreille). Type species Eumenes femoratus Schulthess, 1910. Original designation.

Zethus Fabricius, 1804, Syst. Piez.: xii, 282. Type species Vespa coeruleopennis Fabricius, 1798. Designated by Latreille, 1810, Con. Gén. Crust. Arach. Ins.: 328, 438.

Didymogastra Perty, 1833, Delect. Anim. Artic. Brasil: 144. Type species Didymogastra fusca Perty, 1833. Monotypic. Lethus (!) Say, 1837, Boston J. Nat. Hist. 1: 387.

Heros Saussure, 1855, Ét. Fam. Vesp. 3: 115 (as division of Zethus), non Heros Haeckel, 1840. Type species Zethus gigas Spinola, 1841 (= Vespa coeruleopennis Fabricius, 1798). Monotypic.

Wettsteinia Dalla Torre, 1904, Gen. Ins. 19: 13. Type species Labus sichelianus Saussure, 1875. Designated by Bohart and Stange, 1965, Univ. Calif. Publ. Ent. 40: 25.

Euzethus Dalla Torre, 1904, Gen. Ins. 19: 14. New name. Laboides Zavattari, 1912, Arch. Naturgesch. 78A(4): 65. Type species Labus sichelianus Saussure, 1875. Designated by Bohart and Stange, 1965, Univ. Calif. Publ. Ent. 40: 25. subg. Zethusculus Saussure, 1855, Ét. Fam. Vesp. 3: 118. Type species Zethus jurinei Saussure, 1852. Designated by Ashmead, 1902, Can. Ent. 34: 205.

subg. Zethoides Fox, 1899, Proc. Acad. Nat. Sci. Philad.: 436. Type species Zethoides smithii Fox, 1899, (non Zethus smithii Saussure, 1855; = Zethus chapadensis Bohart and Stange, 1965). Monotypic.

Baeoprymna Cameron, 1912, Timehri 2: 225. Type species Baeoprymna rufoornata Cameron, 1912 (= Zethus miniatus Saussure, 1858). Monotypic.

Protozethus Bertoni, 1926, Rev. Soc. Cient. Paraguay 2: 75. Type species Zethus olmecus Saussure, 1875. Original designation. 
subg. Madecazethus Soika, 1979, Boll. Mus. Civ. Ven. 30: 20, 53. Type species Labus madecassus Schulthess, 1907. Original designation.

\section{Nomina dUbia IN EUMENINAE}

Eumenestiferus Meunier, 1888, Nat. Sicil. 7: 300. Type species Eumenestiferus brasiliensis Meunier, 1888. Monotypic. Unidentified.

Micragris Saussure, 1855, Et. Fam. Vesp. 3: 158 (as division of Synagris Latreille). Type species Synagris spinolae Saussure, 1855. Monotypic. Unidentified.

\section{NOMINA NUDA IN EUMENINAE}

Allepipona Bluethgen, 1951, Mitt. Munch. Entomol. Ges. 41: 194. Antalastoroides Saussure, 1856, Ét. Fam. Vesp. 3: 328 (hypothetical group).

Austrodynerus Soika, 1958 (1957), Boll. Mus. Civ. Ven. 10: 119. Lissodynerus Soika, 1974 (1973), Boll. Mus. Civ. Ven. 24: 119.

\section{ACKNOWLEDGEMENTS}

I thank J. van der Vecht for critically reviewing the manuscript. An earlier version was submitted to Cornell University as part of a doctoral dissertation (Carpenter, 1983). G. C. Eickwort, D. M. Bates, J. L. Cisne and Q. D. Wheeler, members of the author's special committee, read and commented upon this version. Arnold S. Menke, U.S. National Museum, provided valuable comments on a later version.

\section{SUMMARY}

A synonymic checklist of the genus-group names in the Eumeninae is provided. Presently, 177 genera with 34 additional subgenera are considered valid. Neodiscoelius Stange, 1979, is newly synonymized with Protodiscoelius, Dalla Torre, 1904; Hypalastoroides depressus Soika, 1969, is synonymized with Odynerus relativus Fox, 1902; the subgenus Cephalastor Soika, 1982, is raised to genus; and type-species are designated for Nesodynerus Perkins, 1901, and Stenolabus Schulthess, 1910. 


\section{Literature Cited}

BLuethgen, $P$.

1938 (1937). Systematisches Verzeichnis der Faltenwespen Mitteleuropas, Skandinaviens und Englands. Konowia 16: 270-295.

Carpenter, J. M.

1981 (1982). The phylogenetic relationships and natural classification of the Vespoidea (Hymenoptera). Syst. Ent. 7: 11-38.

1983. Phylogenetic studies in Vespoidea (Hymenoptera). PhD Thesis, Cornell University.

Carpenter, J. M. and J. M. Cumming.

1985. A character analysis of the North American potter wasps (Hymenoptera: Vespidae; Eumeninae). J. Nat. Hist. 19: 877-916.

Dalla TORre, K. W.

1904. Vespidae, Gen. Ins. 19: 1-108.

INTERNATIONAL COMMISSION ON ZOOLOGICAL NOMENCLATURE.

1965. Opinion 747. Rygchium Spinola, 1806 (Insecta, Hymenoptera): Validation of emendation of Rhynchium. Bull. Zool. Nomencl. 22: 186-187.

1970. Opinion 893. Eumenidae names of Saussure (Hymenoptera): Grant of availability to certain names proposed for secondary divisions of genera. Bull. Zool. Nomencl. 26: 187-191.

1985. Opinion 1363. Ancistroceroides Saussure, 1855 (Insecta, Hymenoptera): Type species designated. Bull. Zool. Nomencl. 42: 353-354.

Krombein, K. V., P. D. Hurd, D. R. Smith and B. D. BurKs.

1979. Catalog of Hymenoptera in America North of Mexico. Smiths. Inst. Press, Washington, D.C. 

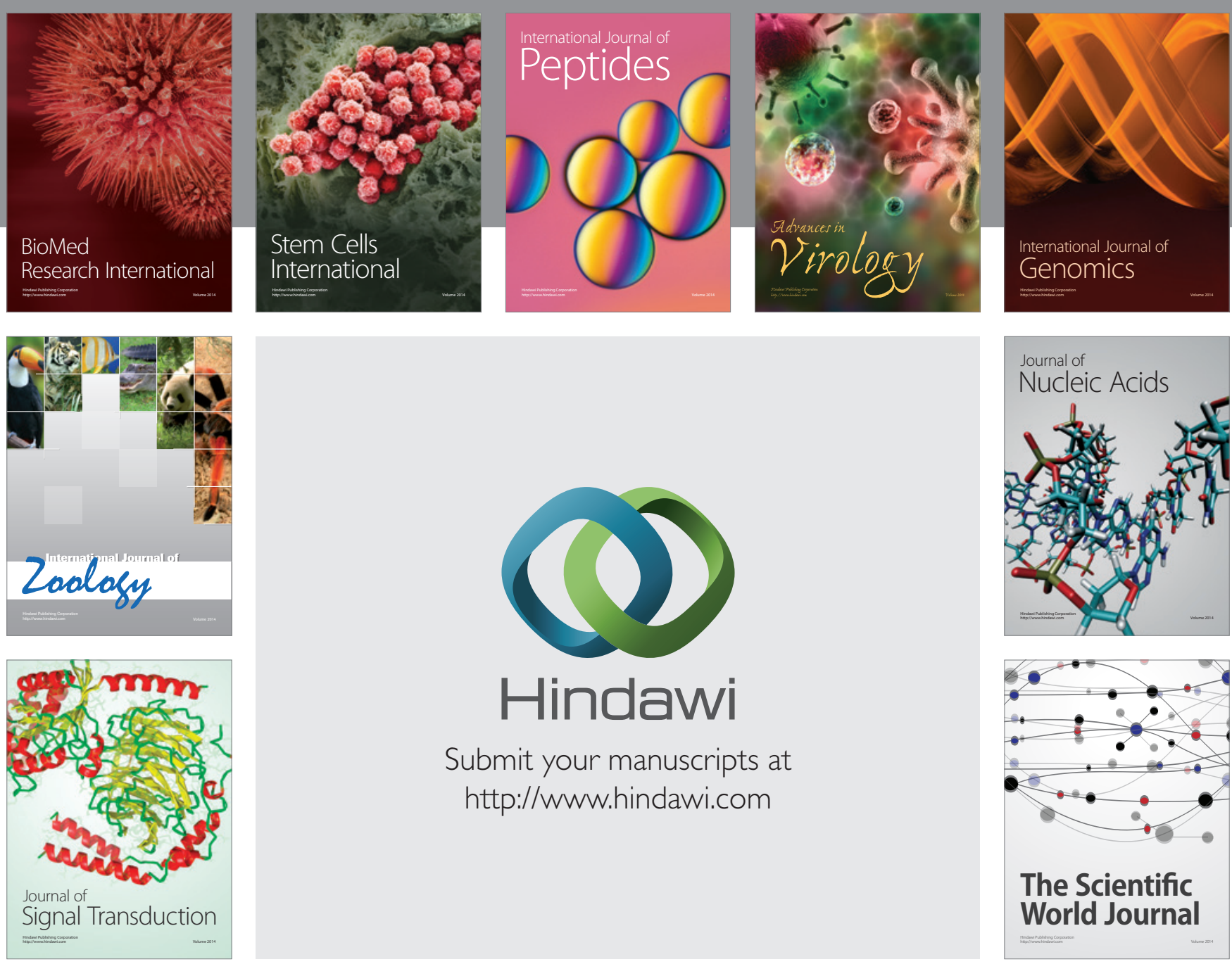

Submit your manuscripts at

http://www.hindawi.com
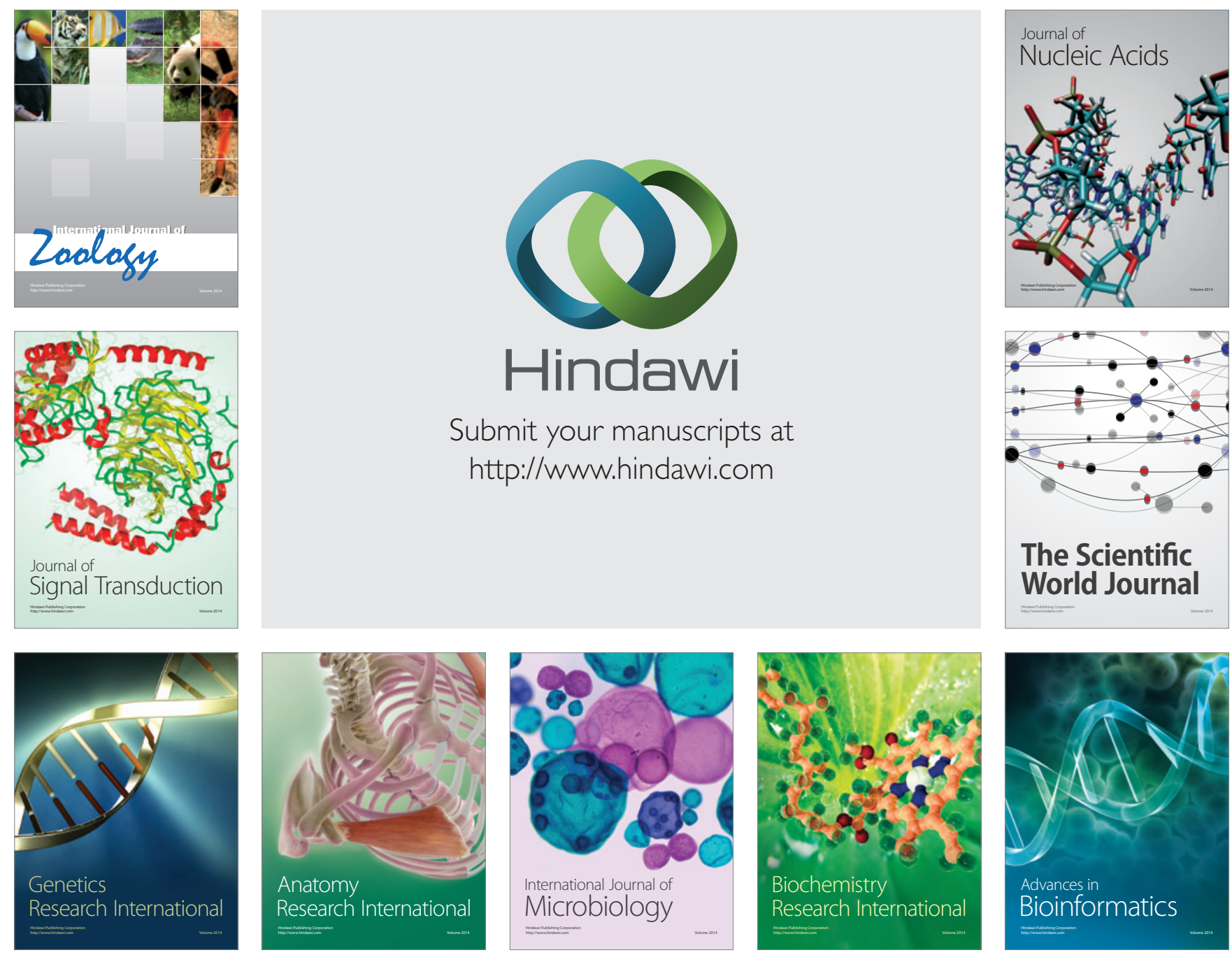

The Scientific World Journal
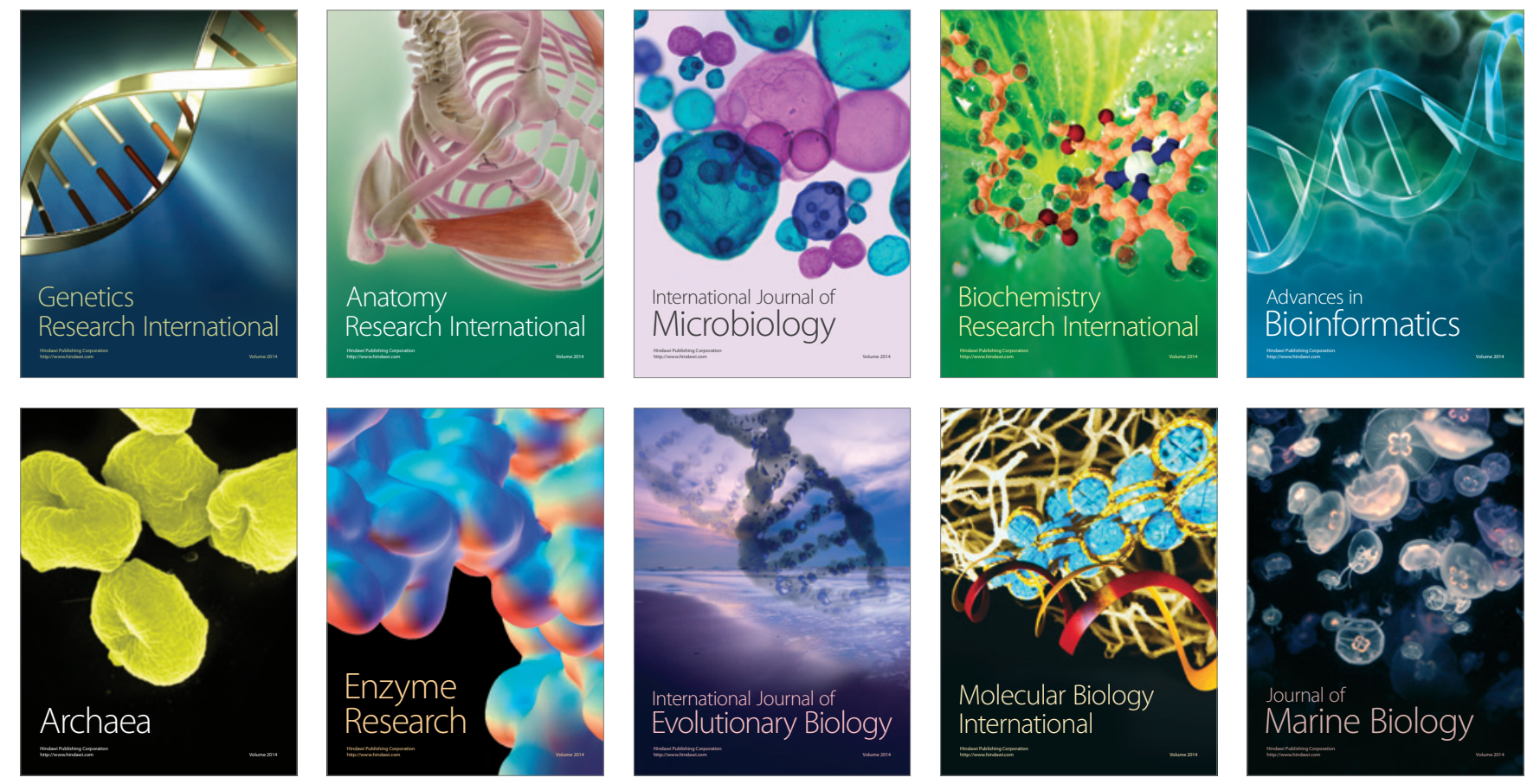